\title{
THE CHANGES OF METABOLIC PROFILE AND ITS ASSOCIATION WITH FAT COW SYNDROME IN HIGH YIELDING COWS DURING DRY PERIOD AND AFTER
}

\author{
Mutlu Sevinc, Veysi Aslan \\ Selçuk University, Faculty of Vet.Med.
}

This investigation was carried out on 17 Brown Swiss cattle in the Central Research Institute of Konya during the winter and lasting until the end of spring. Blood samples were taken from the jugular vein during the 7 th and 8 th month of gestation and after birth and on the 15 th day after parturation. Blood gas analyses and other chemical parameters were measured by using a Blood Gas Analyzer and Autoanalyzer Instruments. At the same time, liver biopsy samples were obtained from each cow during the experiment in order to observe any fat infiltration to the liver.

Blood-ionized calcium (ICa) and normalized calcium (norm $\mathrm{Ca}$ ) concentrations decreased significantly at parturation. The same parameeters were significantly elevated on the 15 th day after parturation. The other metabolic profiles that were measured changed in significantly during the experiment. Fat infiltration to the liver was observed in a few cows but the level of fat infiltration was not severe.

\section{CONCENTRATE FEEDING STRATEGY IN LACTATING DAIRY COWS: METABOLIC AND ENDOCRINE CHANGES WITH EMPHASIS ON LEPTIN}

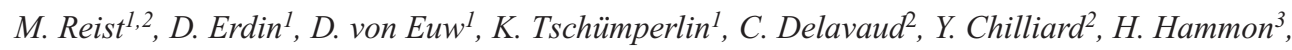 \\ C. Morel ${ }^{3}$, Ch. Philipona ${ }^{3}$, Y. Zbinden ${ }^{3}$, N. Künzil ${ }^{1}$ and J. W. Blum ${ }^{3}$
}

${ }^{1}$ Inst. of Animal Science, Group of Animal Breeding, Swiss Federal Inst. of Technology, CH-8092 Zurich, Switzerland, ${ }^{2}$ Herbivore Research Unit, National Institute for Agricultural Research (INRA), F-63122 St-Genès-Champanelle, France, and ${ }^{3}$ Div. of Animal Nutrition and Physiology, Inst. of Animal Genetics, Nutrition and Housing, Univ. of Berne, CH-3012 Berne, Switzerland.

Relationships between leptin and metabolic, enzymatic, endocrine and zootechnical traits were assessed in 90 multiparous Holstein cows [mean 9'500 kg energy-corrected milk/305 d], held in a research farm from wk 2 ante partum (a.p.) to wk 20 post partum (p.p.). Concentrates ( $8 \mathrm{MJ} \mathrm{NEL/kg}$ $\mathrm{DM}$ ) were fed by automatic feeders for $24 \mathrm{~h}$ at $30 \%$ (C30) or $50 \%$ (C50) of total dry matter (DM) intake (DMI) from wk 1-10 p.p. and were then continuously lowered to $2.4 \%$ and $4 \%$ until wk 34 , resp.. Roughage (6 MJ NEL/kg DM) was fed ad libitum. DMI was measured continuously during 24 h. Milk yield and body weight (BW) were determined twice/d. Milk composition was determined 4 times/wk and milk acetone (AC) was measured weekly. Blood samples for the determination of metabolites, hormones, enzymes and electrolytes were obtained in wk 2 a.p. and weekly from wk 116 and in wk 20 p.p. between 0730 and 0900. Body condition scores (BCS) and ultrasonic measurements of backfat thickness, long. dorsi muscle diameter and fat depth in the pelvic area were evaluated a.p. and in wk 1, 4, 8, 12, 16 and 20 p.p.. In wk 4 p.p., metabolic and endocrine profiles were 
studied in 10 cows of C30 and C50, resp., by hourly blood sampling from 0730-1530. Metabolites and enzymes were measured photometrically and hormones were determined by species-specific radioimmunoassays. Results: Roughage intake was higher, but total DMI was considerably lower in C30 than C50. Peak milk production (42.1 and $43.8 \mathrm{~kg} / \mathrm{d}$ in C30 and C50, resp.) was reached in wk 4 p.p., but mean yields of C30 cows were only $1.28 \mathrm{~kg} / \mathrm{d}$ lower than of C50 cows. Energy balance (EB) was positive a.p., reached a nadir at wk 2 p.p. (-46.0 and -29.6 MJ NEL in C30 and C50, resp.) and then increased up to wk 20 p.p.. Metabolic stress was more marked in C30 than C50 cows, expressed by lower levels of glucose, leptin, insulin, IGF-1, $\mathrm{T}_{3}$, milk protein and lactose, but higher levels of NEFA, BHBA, GH and milk AC, and a faster decrease of BCS and backfat thickness in C30 than $\mathrm{C} 50$ cows over the first 20 wk p.p. $(\mathrm{P}<0.05)$. Blood BHBA and milk AC concentrations peaked in wk 4 p.p.. Enzyme activities were higher in C30 than $\mathrm{C} 50$ cows over the first 8 wk p.p.. Concentration of $\mathrm{Ca}$ was higher and of $\mathrm{Cl}$ lower in $\mathrm{C} 30$ than $\mathrm{C} 50$ cows. The measure of determination of the linear mixed-effects model to assess relationships between leptin and metabolic, enzymatic, endocrine and zootechnical traits was high $\left(\mathrm{R}^{2}=0.85\right)$. However, for leptin the individual cow accounted for most of the variance $\left(\mathrm{R}^{2}=0.61\right)$. Leptin concentrations were positively associated with $\mathrm{BCS}, \mathrm{EB}$, $\mathrm{BW}$, cholesterol, albumin, insulin and IGF-1, and negatively with total DMI and $\mathrm{T}_{3}$, and were higher in cows calving in spring than in fall, i.e. leptin was positively correlated $(\mathrm{P}<0.001)$ with daily photoperiod $(r=0.26)$, mean daily light intensity $(r=0.33)$ and mean daily ambient temperature $(r=0.33)$. In conclusion, leptin is one of several traits which are connected with $\mathrm{EB}$, but it is also related to other factors.

\title{
RESPONSE TO INTRAVENOUS GLUCOSE CHALLENGES IN DAIRY COWS FED DIFFERENT AMOUNTS OF A TOTAL MIXED RATION IN THE DRY PERIOD
}

\author{
Kjell Holtenius ${ }^{1,2}$ and Sigrid Agenäs ${ }^{2}$ \\ ${ }^{1}$ Department of Ruminant Medicine and Veterinary Epidemiology, ${ }^{2}$ Department of Animal Nutrition \\ and Management, Swedish University of Agricultural Sciences, Uppsala, Sweden.
}

The feeding regime during the dry period has often a marked impact on the metabolism during the post-parturient period in dairy cows. In the present study the response to intravenous glucose challenges was tested in cows given different amounts of a total mixed ration (TMR) during the dry period. Multiparous high yielding cows $(\mathrm{N}=24)$ were divided into three equal groups. The groups were offered TMR corresponding to 70, (Low intensity) 110 (Medium intensity) or 170 (High intensity) MJ ME/day during the eight weeks dry period. After parturition all cows were offered an other TMR ad libitum during the first twelve weeks. The energy in the TMR offered during the dry period contained 11.8 MJ ME/kg DM and the TMR offered during the lactation contained 12.2 MJ ME/kg DM. Feed intake, body weight and body condition scoring was monitored during the experiment. Blood was regularly analysed for key metabolites and hormones. Glucose challenges were performed three weeks prepartum and three weeks postpartum. The cows were injected with $150 \mathrm{mg}$ glucose $/ \mathrm{kg}$ body weight in the jugular vein. Blood was then collected frequently for one hour. The rate of glucose dis- 
appearance prior to parturition was $2.4,2.7 \& 4.1 \%$ /min for the low, medium \& high intensity fed groups respectively. The high intensity fed cows were markedly hyperinsulinemic. However, the insulin response to the glucose challenge, calculated as the area under the response curve (AUC), did not differ between the 3 treatments. Cows which were subjected to the high intensity feeding during the dry period showed a pronounced reduction in feed intake and a deeper nadir of negative energy balance after parturition. In cows which were fed the low and medium intense dry period diets the glucose disappearance rate increased with $150 \%$ as compared to the dry period. The increase was much less, about $30 \%$, in cows fed the high intensity diet during the dry period. The insulin response to the glucose challenge was reduced to one third of the response prior to parturition regardless of treatments in the dry period.

These results suggest that the glucose disappearance rate is strongly influenced by the level of feed intake. The pancreatic insulin response to glucose was markedly reduced in the post-partal period in all three treatments. Overfeeding in the dry period led to a deeper and longer period of negative energy balance post partum but the pancreatic insulin response to glucose was not affected.

\title{
OBJECTIVE MEASUREMENT OF HEALTH IN PIGS - APPLICATION OF ACUTE PHASE PROTEINS
}

\author{
Jens Peter Nielsen and Henrik Hagbard Petersen \\ Royal Veterinary and Agricultural University, Copenhagen, Denmark, jpni@kvl.dk
}

In this presentation health is defined as absence of disease in individual animals or populations. High health status indicates that no or only mild disease is present. Low health status indicates that several or severe diseases are present.

Measurement of health status. Objective measurement of health status is difficult. Serological or microbiological testing for a number of specific infections is possible, but often impractical. Clinical examination may reveal clinical disease problems but do not reveal sub-clinical disease an may suffer from lack of objectivity. Sub-clinical disease may be measured indirectly by recording of growth rate, feed consumption or reproductive performance. However, these indicators are difficult to interpret. Pathological findings at slaughter only give a prevalence estimate and data-quality may be poor. Acute phase proteins are produced as a non-specific response to inflammation, trauma and infection in animals and may be measured objectively in serum samples.

Why measure health status? Objective measurement of health status or disease severity is important in several situations. It creates an important research tool when studying pathogenesis and inflammatory processes in experimental animals or when testing the efficacy of pharmaceuticals or comparing their effect in experimental or field trials. Objective measurement of health status allows comparison of health in different production. Categorisation of herds in health levels may be relevant in the future.

Acute phase proteins in pigs. Acute phase proteins are an important part of the acute phase response following tissue damages. The damage may be induced by infections, toxins, trauma etc. and results 
in liberation of inflammatory mediators into the systemic circulation. These mediators include cytokines such as IL-1, TNF, IL-4, IL-6, IL-8, IL-10 and IL-11. The cytokines induce increased (positive) or decreased (negative) hepatic synthesis of serum acute phase proteins. Several porcine infections has been shown to mount an acute phase response e.g. Actinobacillus pleuropneumoniae, toxigenic Pasteurella multocida, Mycoplasma hyorhinis, PRRS virus etc. The major acute phase proteins in pigs include haptoglobin, major acute phase protein (MAA), serum amyloid A (SAA), alfa1 acid glycoprotein (AGP) and C-reactive protein.

Serum haptoglobin in relation to clinical signs and production systems. In a case-control study in 14 pig herds including serum samples from 170 healthy and clinically diseased pig pairs. A significant increase in haptoglobin concentrations was found in pigs with clinical signs such as respiratory disease, lameness, ear and tail biting and diarrhoea. In another study 7 herds free from 5 specific pathogens (Danish SPF-herds) were compared to 4 conventional herds suffering from some or all of the infections absent in the SPF herds. In each herd serum samples were taken from young, medium or old finishing pigs. At the same time the animals were subjected to a standardised clinical examination. The study showed that finishing pigs from SPF herds had a significantly lower haptoglobin serum concentration than pigs from conventional herds. However, the variation between SPF herds was high, indicating that SPF-production is not always equivalent to high health status.

\title{
HAPTOGLOBIN AS AN INDICATOR FOR ANIMAL WELFARE: EFFECTS OF DIFFERENT HYGIENIC CONDITIONS AND TRANSPORT STRESS ON HAPTOGLOBIN PLASMA CONCENTRATION
}

\author{
Gymnich, S. ${ }^{1}$, S. Knura-Deszczka, ${ }^{1}$ K. Wimmers, ${ }^{2}$ M. Bidlingmaier, ${ }^{3}$ K. Schellander, ${ }^{2}$ B. Petersen ${ }^{1}$ \\ ${ }^{1}$ Department of Anatomy, Physiology and Hygiene of Domestic Animals, Katzenburgweg 7-9, 53115 Bonn, \\ Germany; ${ }^{2}$ Department of Animal Breeding, Bonn, Germany; 4Medical Center, München, Germany
}

The aim of the present work was to examine the suitability of the acute-phase-protein (APP) haptoglobin ( $\mathrm{Hp}$ ) as an indicator of animal welfare represented by different hygienic conditions and transport stress.

2 breeder-fattening farms with different hygienic conditions recorded by specific checklists were examined by monitoring 2 indicator groups of 16 pigs from each farm. Haptoglobin determination was performed 4 times by blood sampling from the end of the rearing period up to 4 weeks after housing, weight gain and clinical symptoms were observed. In contrast to farm A, farm B only acquired half of the maximally available points concerning relevant hygiene factors and was therefore rated "unsufficient". The pigs of farm B showed more often clinical symptoms than those of farm A which was reflected by comparing the time course of growth of the two fattening groups. The performance of pigs in farm A followed the normally observed physiological course of growth while the growth of pigs in farm B was depressed. It were conspicuous that there was also statistically significant differences in Hp-concentration between animals showing no observable clinical deficits from farm B in comparison to pigs from farm A. 
The results showed that the acute phase protein haptoglobin is a very sensitive parameter that can support the evaluation of the general health status of fattening pigs.

To examine the suitability of the parameter haptoglobin as an indicator for transport stress the course of the haptoglobin plasma and cortisol saliva concentrations in 93 fattening pigs was monitored for four days at weights of 30 and $80 \mathrm{~kg}$. At the beginning of the study pigs were randomly assigned to one of three test protocol groups:

- Negative control group: no treatment

- Transport group: 3 hours transport

- Myostress group: injection of myostress, which is normally used for testing stress with the creatine-kinase-test.

To observe if animals having haptoglobin levels above or below the cut-off value of $0.5 \mathrm{mg} / \mathrm{ml}$ react differently to stress the haptoglobin measurements were divided into 2 groups - those above 0.5 $\mathrm{mg} / \mathrm{ml}$ and those below this value. The experimental treatment did not significantly influence the haptoglobin levels of the examined animals but significant changes because of the health status could be observed. The parameter cortisol did however show a significant increase in both 30 and $80 \mathrm{~kg}$ pigs after transport.

This results reveal that transport stress can be monitored by evaluating cortisol levels whereas haptoglobin does not appear to be a significant parameter for measuring transport stress but as an sensitive indicator for the health status of fattening pigs. Therefore haptoglobin can be a valuable tool within the scope of supplier assessment.

\title{
HAPTOGLOBIN IN PIGS: DEVELOPMENT AND VALIDATION OF AN ENZYME IMMUNOASSAY FOR VARIOUS BODY FLUIDS AND ESTABLISHMENT OF PHYSIOLOGICAL REFERENCE LEVELS
}

\author{
Stephanie Hiss ${ }^{1,2}$, Mark Hennies ${ }^{1}$, Stefanie Gymnich ${ }^{1}$, Brigitte Petersen ${ }^{1}$ \\ and Helga Sauerwein ${ }^{1}$ \\ ${ }^{1}$ Institute for Anatomy, Physiology and Hygiene of Domestic Animals, Katzenburgweg 7-9,53115 Bonn, \\ Germany; ${ }^{2}$ Biofocus GmbH, Recklinghausen, Germany
}

Haptoglobin (Hp) is an acute phase protein. Quantifying its concentrations is presently discussed as being useful to monitor animal health and welfare. To obtain a simple, rapid and robust assay system we developed a competitive enzyme-immuno assay which is species specific and also sensitive enough to be applied in matrices in which low concentrations are to be expected, e.g. in saliva.

$\mathrm{Hp}$ was purified from porcine serum after sodium sulphate precipitation by affinity chromatography on hemoglobin crosslinked to sepharose followed by gel filtration. Purity and identity were confirmed by SDS-PAGE. The purified protein was used to immunise rabbits. In all antisera obtained, the titres were determined and the specificity was evaluated by Western blotting. In the assay biotinylated porcine (p) Hp was used as tracer and was incubated together with either Hp standard or sample in microtiter plates coated with anti rabbit sheep IgG Fc fragments. After adding the specific 
rabbit antiserum, plates were incubated for 1 hour, washed and evaluated via a streptavidin peroxidase system with TMB as substrate. The limit of detection was $0.02 \mathrm{mg} / \mathrm{L}$, parallelism of serum dilutions was proven and recovery of $\mathrm{pHp}$ added to serum samples was $99.6 \%$. The coefficients of intra and inter assay variation were $3.3(n=5)$ and $10.2(n=16)$, respectively. Comparing the new assay with an established nephelometric assay system using antibodies against human Hp (Lipperheide et al., 1998), the concentrations measured in 86 serum samples were identical in both assays $(\mathrm{r}=0,96$, $\mathrm{p}<0.01$ ). In contrast to biochemical or nephelometric systems, there is no interference in haemolytic or turbid samples in our assay and significantly lower amounts of antiserum are needed. In addition, it can validly be applied in blood serum, plasma, whole blood, saliva and in meat juice, the latter being possibly of particular relevance for slaughter line controls.

$\mathrm{Hp}$ was measured in blood sera from male and female pigs at various physiological stages. Pigs from private and from experimental farms were clinically inspected and diagnosed as healthy. The following ages were monitored: suckling piglets ( 2 weeks old, $n=10)$, weaned piglets ( 4 weeks, $n=10$ ), fattening pigs ( 6 months, $n=10)$, gilts $(n=10)$ and sows $(n=14)$, gilts $(n=6)$ and sows $(n=19)$ within 14 days after conception, sows being pregnant for 2 months $(n=30)$, sows being pregnant more than 100 days $(n=6)$ and lactating sows $(n=24)$.

Hp concentrations were lower in suckling piglets than in weaned or fattening pigs. In sows from different reproductive stages, highest concentrations were observed in lactating and non-pregnant sows. The assay presented herein provides a powerful tool to monitor Hp concentrations in various body fluids irrespective of sample condition. Based on the reference values for blood provided herein, it can be effectively used to control health status at the different stages of pig production.

Lipperheide, C., N. Diepers, F. Lampreave, M. Alava \& B. Petersen (1998), J. Vet. Med. A 45, 543-550

\title{
HAPTOGLOBIN RESPONSES TOWARDS VACCINATION WITH THREE DIFFERENT ANTIGENS IN PIGS
}

\author{
Michelle Rekitt ${ }^{1,2}$, Stephanie Hiss ${ }^{1,2}$, Dorothee Dickhöfer ${ }^{1}$, Klaus Wimmers ${ }^{3}$, Karl Schellander ${ }^{3}$, \\ Brigitte Petersen ${ }^{1}$ and Helga Sauerwein ${ }^{1}$
}

${ }^{1}$ Institute for Anatomy, Physiology and Hygiene of Domestic Animals, Katzenburgweg 7-9, 53115 Bonn, Germany, ${ }^{2}$ Biofocus GMBH, Recklinghausen, Germany, ${ }^{3}$ Institute for Animal Breeding Science, Bonn, Germany

Haptoglobin (Hp), an acute phase protein, is elevated in animals exposed to immunological burdens. In order to be able to use Hp concentrations in blood as an indicator for animal health in general, the potential changes induced by common vaccinations need to be characterised and eventually be considered. We therefore recorded $\mathrm{Hp}$ serum concentrations in pigs before and after vaccinations against the porcine respiratory and reproductive syndrome (PRRS), against Aujeszky's disease (AD) and mycoplasma hyopneumonia (Myc).

PRRS vaccination was done in 25 piglets one day after weaning at 4 weeks of age and blood samples for $\mathrm{Hp}$ analyses were collected on day 0 and 28 from all animals and on days 7, 14 and 21 from 4 an- 
imals. PRRS titres were determined in day 0 and day 28 samples. In addition, 10 sows being pregnant for 6 weeks were monitored after a PRRS booster injection. Blood samples were taken on day $0,1,2,3,4,7,10,14,21$ and 28. Hp concentrations and PRRS titres were determined in all samples. Vaccinations against AD and Myc were done in F2-offsprings of an experimental population based on the reciprocal cross of Berlin Miniature Pig and Duroc. 150 piglets being 7 weeks old were immunised against Myc; at 14 weeks of age these animals were then immunised against AD. Blood samples were taken on day 0 before and day 7 after the vaccinations. The same antigens were used in another trial in which the experimental design was identical except that control groups were included: for Myc, 28 pigs were immunised and 19 pigs served as controls. AD vaccination was done in 23 pigs with 17 control pigs.

Hp concentration in piglets was increased after immunisation against PRRS on day 7 and 14. Compared to the initial baseline concentrations, 10-fold and 8-fold higher values were observed, respectively. 11 out of the 25 piglets were PRRS titre positive before vaccination, but there was no difference between basal Hp concentrations in these animals compared to the PRRS negative ones. Hp response between day 0 and day 7 was not related to the extent of titre increases between day 0 and day 28. In sows, Hp concentrations were increased until day 4 after vaccination. The maximal Hp concentrations were 1.5-fold higher than prevaccination levels. After day 7 baseline values were reached again. PRRS titres in sows were increased on days 21 and 28. Following vaccination against Myc or AD, Hp concentrations in pigs were consistently increased.

Vaccination against viral and bacterial antigens uniformly led to an increase of Hp concentrations. The extent of the elevations observed showed no perceptible relation to the antigen used. In conclusion, increased haptoglobin serum concentrations have to be expected when animals are vaccinated. Efforts towards characterisations of animal health by means of haptoglobin measurements therefore will have to consider anamnesis to be able to differentiate between increases of haptoglobin induced by either vaccination or by immunological burdens.

\section{THE POSITION OF THE ABOMASUM IN THE FIRST SIX WEEKS AFTER CALVING}

\section{Steven C.L. van Winden}

Department of Farm Animal Health, Division Ruminant Health, Faculty of Veterinary Medicine, Utrecht University, The Netherlands. Visiting address: Yalelaan 7, 3584 CL Utrecht. Postal address: PO box 80.151, 3508 TD Utrecht, The Netherlands

In the highly pregnant cow the abomasum is forced to the cranial and ventral part of the abdomen by the gravid uterus. After calving, the abomasum is located at the left ventral part of the abdominal cavity. This mechanism is assumed to be one of the factors contributing to the pathogenesis of abomasal displacement (AD).

In the present investigation, the changes of the position of the abomasum were studied in 6 HolsteinFriesian cows by transabdominal ultrasonography in the first 6 weeks after calving. The position of the abomasum was determined via measuring the distance between the abomasal wall being visible 
by examination of the left abdominal wall and the ventral midline. (The precision of this measurement had been determined earlier by examination of 5 additional cows in intervals during a 24-hour period). Samples of rumen juice were taken via a Sørensen-Schambye probe for determination of the $\mathrm{pH}$-value and the osmotic pressure.

The reproducibility of the measurements was determined via calculating the coefficient of variation. The position of the abomasum in relation to the number of days post partum, feed intake and the $\mathrm{pH}-$ value and osmotic pressure of the rumen fluid was estimated via regression analysis. After the analysis, a backward stepwise linear regression was performed to determine combined influences on the position of the abomasum.

A circadian, diurnal variation was observed in the position of the abomasum. After correcting for the circadian variation, a 0,136 coefficient of variation was determined for the method of measurement of the position of the abomasum.

With respect to the left body wall, the position of the abomasum was highest in the first days after calving; the height declined during the following weeks. The abomasum was found to form a blind pocket near the left body wall.

Feed intake, $\mathrm{pH}$-value and osmotic pressure of rumen juice influenced the position of the abomasum. After backward stepwise linear regression the influence of the feed intake was not significant, since feed intake was closely related with the number of days postpartum.

The results show that ultrasonography is a good non-invasive method for determining the position of the abomasum. Measurements of the position of the abomasum are reproducible. The presence of a blind pocket formed by a part of the abomasum is more evident when the organ has a high position. There is a relation between the number of days after calving, $\mathrm{pH}$-value and osmotic pressure of the rumen juice and the position of the abomasum.

These findings support the hypothesis that the shift of the abdominal organs after calving could form an important factor in the pathogenesis of $\mathrm{AD}$. The presence of the abomasum on the left side of the abdominal cavity after calving and the declining height with time could explain why AD to the left side preferentially occurs within the first weeks after calving.

\title{
THE ANTI-OXIDATIVE STATE BEFORE AND AFTER A DIAGNOSED ABOMASAL DISPLACEMENT IN COWS
}

\author{
M. Fürll, M.N. Dabbagh, H. Kirbach, C. Nauruschat, T. Sattler, H. Wilken \\ Department of Internal Medicine, Veterinary Faculty Leipzig; \\ e-mail: mfuerll@rz.uni-leipzig.de
}

\begin{abstract}
Objective
Abomasal displacement (DA) is one of the most important non-infection diseases in high yielding dairy cows. Aetiological factors are mainly disturbances in energy metabolism beginning in part in the dry period. Oxidative stress is involved in the pathogenesis of this disease. But there is a lack on knowledge's.
\end{abstract}




\begin{abstract}
Aims
In three complexes investigations were performed to answers to the following questions: 1) Significance of antioxidants in the aetiology of abomasal displacement, 2) the antioxidative state at the time of diagnosis of DA, 3) development of antioxidative state after reposition of displaced abomasum.
\end{abstract}

\title{
Experimental design
}

The antioxidative state was checked in 20 healthy and 137 cows suffering from abomasal displacement: 1) 8 cows on 3rd day after parturition (ap) before DA and at the diagnosis of DA; 2) 87 cows at the diagnosis of DA; 3) 21 cows before and in the course of 24 hours after DA reposition; 4) 21 cows before as well as on first and third day after DA reposition. In the blood were analysed superoxide dismutase (SOD, erythrocyte lysate resp. serum), glutathione peroxidase (GPX, heparinized whole blood), Trolox Equivalent of Antioxidative Capacity (TEAC, serum), CK and AST (serum) as well as further haematological and clinical chemical parameters (Hitachi 704, Technicon 1).

\section{Results}

The SOD-activity increased from $6729 \pm 1048 \mathrm{U} / \mathrm{ml}$ one week (w) ap to $7506 \pm 1208 \mathrm{U} / \mathrm{ml}$ four w ap in erythrocyte lysate (el) of healthy cows resp. from $10 \mathrm{U} / \mathrm{ml}(0 / 50) 140 \mathrm{U} / \mathrm{ml}(270 / 340)$ in serum. The same trend was observable in TEAC with $226 \pm 42$ to $310 \pm 30 \mu \mathrm{mol} / 1$. At the second day ap were not TEAC differences between healthy and cows with later DA $(237 \pm 37 \mu \mathrm{mol} / 1)$ as well as at the diagnosis of DA $(250 \pm 74 \mu \mathrm{mol} / 1)$. Cows with DA had significant higher CK activities at second day ap (416 U/1[157/771]) and at the diagnosis of DA (313 U/1 [166/414]). The SOD activity was in 87 cows at diagnosis of DA $7125 \mathrm{U} / \mathrm{ml}$ el (6154/ 8625) and did not differ from healthy cows. Cows with left side DA had slightly lower SOD activities than cows with right side DA. 44,8\% of the cows had SOD activity $<7000 \mathrm{U} / \mathrm{ml}$ el. They had higher CK and ASAT activities, bilirubin and BHB and lower cholesterol concentrations $(\mathrm{p}<0,05)$. Cows with SOD activities $<7000 \mathrm{U} / \mathrm{ml}$ el therefore suffered from massive metabolic stress. The GPX activity in cows with DA was $385 \pm 103 \mathrm{U} / \mathrm{mg} \mathrm{Hb}$. Between SOD and GPX was found a negative correlation $(\mathrm{r}=-0,40, \mathrm{p}<0,01)$. In cows with low GPX-activity the bilirubin concentrations were low, while in cows with high GPX-activities these concentrations were greatly increased. After reposition of left side DA SOD activities decreased shortly, in contrast in cows with right side for more than $24 \mathrm{~h}(\mathrm{p}<0.05)$. Cows with left side DA had higher TEAC concentrations compared with right side DA. After reposition of DA the TEAC concentration didn't differ significantly.

\section{Conclusions}

The antioxidative capacity (SOD, TEAC) increases in healthy cows after parturition. The TEAC concentration is not changed before DA, but the CK activity is higher ( $<<0.05)$. Approximately $45 \%$ of the cows with DA have a stressed antioxidative system (SOD-activity $<7000 \mathrm{U} / \mathrm{ml} \mathrm{el}$ ). This is mostly the case in cows with longer existent moderate stress, such as left side DA. Shorter and massive metabolic stress (bilirubin $>17 \mathrm{mmol} / 1$, glucose $>8,8 \mathrm{mmol} / \mathrm{l}$ ) is equivalent to GPX-activities $>500$ $\mathrm{U} / \mathrm{mg} \mathrm{Hb}$. In these cases the SOD activity is only slightly decreased. The SOD activities decrease special after reposition of right side DA. 